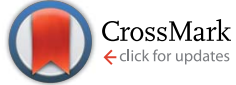

Cite this: RSC Adv., 2017, 7, 3736

Received 24th October 2016 Accepted 24th December 2016

DOI: 10.1039/c6ra25750j

www.rsc.org/advances

\section{Recrystallization of copper at a solid interface for improved CVD graphene growth $\dagger$}

\author{
Ya-Ping Hsieh, ${ }^{\text {*a }}$ Ding-Rui Chen, ${ }^{a}$ Wan-Yu Chiang, ${ }^{a}$ Kai-Jyun Chen ${ }^{b}$ \\ and Mario Hofmann ${ }^{b}$
}

The quality of CVD-grown graphene is intimately linked to the morphology of the employed catalyst. One commonly employed route to enhanced catalyst quality is recrystallization prior to graphene growth. The dimensions of a catalyst's single-crystalline domains, however, are limited by the stability of dislocations at the grain boundaries. We here employ a solid material in contact with the catalyst as a sink for dislocations. It was found that the interaction between $\mathrm{Cu}$-foil and a solid cap significantly alters the recrystallization kinetics and achievable grain size. This development originates from an improved strain-relaxation of up to $1 \%$ during recrystallization which can support the formation of commonly unstable surface orientations. Correlation of diffraction measurements and atomic force microscopy reveals a direct impact of the strain relaxation on the decrease in copper surface roughness. Finally, we demonstrate the improvement of the quality of graphene grown on thus prepared foils through spectroscopic and carrier transport measurements.

\section{Introduction}

Graphene is a two-dimensional nanomaterial that has been heralded as an enabling material for novel electronic devices, sensors, and transparent conductors. ${ }^{1}$ To fulfill requirements in these areas, the increase in graphene quality and crystallinity synthesized by chemical vapor deposition (CVD) is the focus of ongoing efforts.

One important aspect that was found to affect graphene's domain size, domain orientation, and carrier mobility, is the crystallinity of the underlying catalyst surface. ${ }^{2-5}$ Previous reports showed that the smoothness of $\mathrm{Cu}$ will dominate the defect density of graphene. ${ }^{6-9}$ Moreover, $\mathrm{Cu}$ grain boundaries act as obstacles to graphene growth ${ }^{\mathbf{1 0 - 1 2}}$ and deteriorate

${ }^{a}$ Graduate Institute of Opto-Mechatronics, National Chung Cheng University, Chiayi, 62102, Taiwan.E-mail: yphsieh@ccu.edu.tw

${ }^{b}$ Department of Material Science and Engineering, National Cheng Kung University, Tainan, 70101, Taiwan.E-mail: Mario@mail.ncku.edu.tw

$\uparrow$ Electronic supplementary information (ESI) available: Detailed characterization of $\mathrm{Cu}$ foil, graphene quality and continuity. See DOI: 10.1039/c6ra25750j graphene's quality. ${ }^{12}$ Therefore, an ideal substrate for high quality graphene growth needs to have larger $\mathrm{Cu}$ grains.

Several studies demonstrated that the grain size of commonly used $\mathrm{Cu}$ foil can be enhanced by annealing prior to CVD growth. ${ }^{\mathbf{1 3 - 1 5}}$ This grain growth occurs due to the presence of large amounts of strain that are introduced during the foil production process. ${ }^{\mathbf{1 6 , 1 7}}$ Grain growth then proceeds through recrystallization which displaces dislocations in the lattice and thus releases the strain. ${ }^{18}$ The grain growth seizes, however, when dislocation motion and grain boundary motion reach an equilibrium. ${ }^{\mathbf{1 9}}$

To overcome this fundamental limitation, we require a method to affect the concentration of dislocations and thus change the equilibrium condition. One such way is the introduction of a sink of dislocations. If dislocations are removed from the grain boundaries, grain growth could proceed further and significantly larger grains are achievable. ${ }^{20}$

We here demonstrate that a solid interface in contact with the $\mathrm{Cu}$ foil can act as such a sink for dislocations. By placing a solid cap onto the $\mathrm{Cu}$ foil, the recrystallization process was found to be significantly altered. Electron back scatter diffraction (EBSD) showed a slower recrystallization process and larger achievable final grain size. This behavior was found to be caused by an enhanced strain relaxation at the $\mathrm{Cu} /$ solid interface. The observed stress relaxation furthermore lowers the roughness of $\mathrm{Cu}$ and ultraflat surfaces with $R_{\mathrm{a}}<0.4 \mathrm{~nm}$ were obtained. Finally, graphene grown on these cap-annealed copper substrates were found to have larger grain sizes and an enhanced carrier mobility.

\section{Experimental}

Graphene was grown on $25 \mu \mathrm{m}$ thick foil copper foil (Alfa Aesar 46365, purity $99.8 \%$ ) following established procedures. ${ }^{21}$

Annealing was carried out using an atmosphere of argon and hydrogen with a volume ratio of $7: 2$ at a pressure of 700 Torr for various times. During annealing, caps of graphite were placed on top of one $\mathrm{Cu}$ foil while an uncapped $\mathrm{Cu}$ foil was exposed to the same annealing process (Fig. 1) and an 
additional weight (quartz, $7 \mathrm{~g}$ ) was place on top of graphite to increase the contact between copper and graphite.

Afterwards, AFM images were taken (FORCE Genie) and the roughness of copper was analyzed using the Gwyddion software package.

The texture and grain boundary distribution of the copper were examined by electron back scattering diffraction (EBSD, JSM 7001F) operating at $20 \mathrm{kV}$. X-ray diffraction was carried out on a Bruker D2 Phaser for more than 4 different samples for each process condition and peak positions were averaged after individual fitting.

Graphene was grown under a flow of $200 \mathrm{sccm}$ hydrogen and $10 \mathrm{sccm}$ methane at 10 Torr following previous reports. ${ }^{21}$ Two different growth durations (20 min and 6 hours) were used to obtain separate grains and well-connected films, respectively.

Mobility of graphene was extracted from field effect transistors (FETs) with channel lengths of $150 \mu \mathrm{m}$ which were fabricated on $\mathrm{SiO}_{2}$-supported graphene by deposition of gold through a shadow mask. The field effect mobility was extracted following previous reports. ${ }^{22}$

To visualize graphene on copper under an optical microscope (OM), graphene-covered copper was first air oxidized following previous report. ${ }^{23}$

\section{Results and discussion}

Electron back scatter diffraction (EBSD) of the pristine $\mathrm{Cu}$ surface shows small size of grains $(\sim 50 \mu \mathrm{m})$ with a predominantly (100) oriented surface and few higher angle textures (Fig. 1(a)).

We find that the grain size of $\mathrm{Cu}$ increases with annealing time in agreement with previous reports. ${ }^{13}$ However, even after $12 \mathrm{~h}$ annealing the grain size is below $200 \mu \mathrm{m}$.

This behavior is in marked difference with samples that were brought in contact with a solid cap and annealed under the same conditions (see Fig. 1(b)). We observe a consistent increase of grain size compared to uncapped annealing under
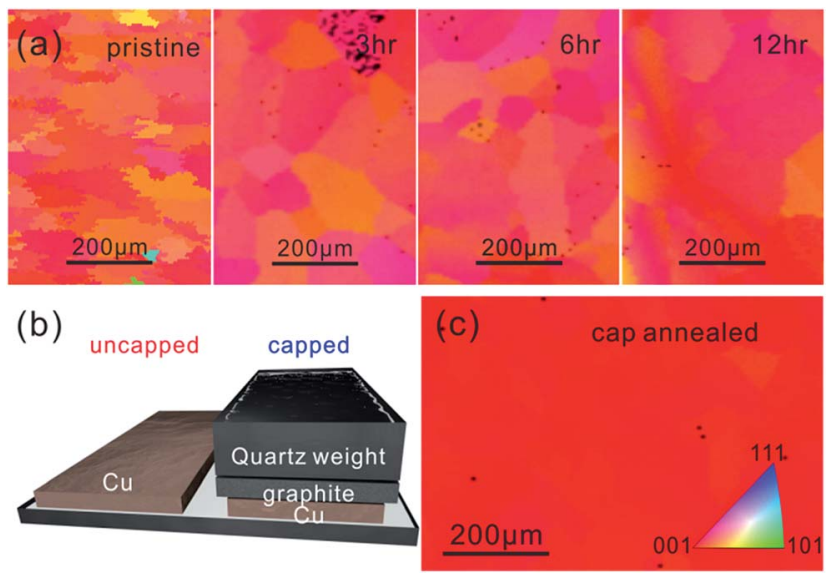

Fig. 1 (a) EBSD images before and during uncapped annealing (b) schematic of capped annealing process, (c) EBSD of $\mathrm{Cu}$ foil after 8 hours capped annealing. identical conditions. After 12 hours annealing most cm-sized samples did not exhibit any grain boundaries indicating complete conversion into single crystals (Fig. 1(c)).

In order to understand this behavior, we carried out 3 hour annealing experiments at different temperatures for capped and uncapped $\mathrm{Cu}$ foil. Fig. 2(a) shows an Arrhenius plot of the grain dimension as a function of inverse temperature extracted from backscattered electron imaging (BEI) (ESI Fig. S1†) and a clear linear trend is observed which suggests a temperature activated diffusion process to dominate the grain growth in both capped and uncapped annealing conditions.

The activation energy was then extracted from the Arrhenius equation according to

$$
K=A \mathrm{e}^{-\frac{E_{\mathrm{a}}}{k_{\mathrm{B}} T}},
$$

where $k_{\mathrm{B}}$ is the Boltzmann constant.

We find that the activation energy of grain growth for uncapped copper foil $(0.45 \mathrm{eV})$ is significantly lower than capped copper foil $(1.2 \mathrm{eV})$. The value of the capped case is close to the values for bulk copper. ${ }^{24}$ A lower activation energy had been previously associated with strain due to grain boundaries. ${ }^{24}$

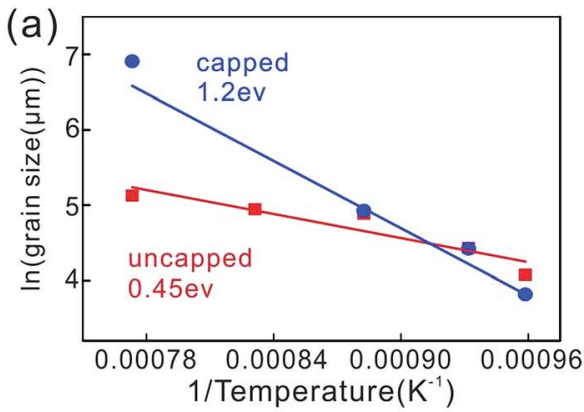

(b)

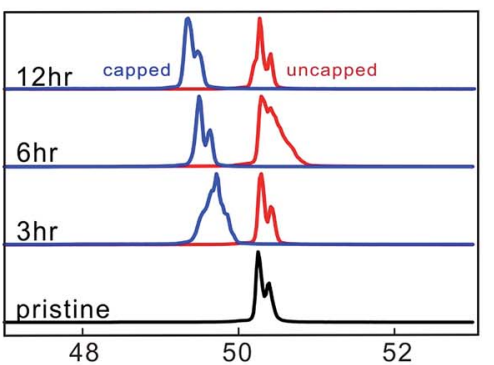

(c)
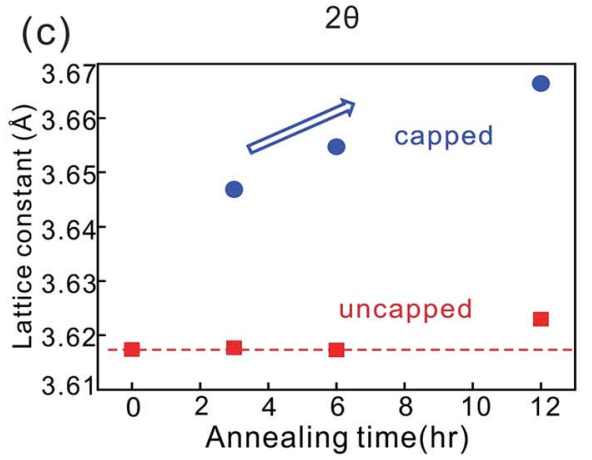

Fig. 2 Comparison of uncapped and capped annealing conditions: (a) Arrhenius plot of grain size (b) overlay of XRD spectra evolution during annealing, (c) extracted lattice spacing vs. annealing time. 
These results suggest that uncapped copper foil exhibits a significantly higher strain whereas capped annealing results in the lowering of film strain due to recrystallization.

X-ray diffraction (XRD) was carried out to elucidate the presence of strain in the $\mathrm{Cu}$ lattice. The main XRD peak associated with the (200) direction exhibits a different behavior for uncapped and capped $\mathrm{Cu}$ foil (Fig. 2(b)). The lattice spacing was extracted from the XRD peak position and we find that the lattice constant is increasing with time for capped $\mathrm{Cu}$ foil but remains constant for uncapped $\mathrm{Cu}$ (Fig. 2(c)). We therefore conclude that the presence of an interface causes a significant relaxation of the $\mathrm{Cu}$ that can reach $1.3 \%$ after $12 \mathrm{~h}$ annealing.

The observed significant decrease of lattice strain during recrystallization could also explain the occurrence of different crystal textures after annealing. We observe that a majority of uncapped $\mathrm{Cu}$ annealed at various times will retain its 100 direction (Fig. 1(a)). This is expected since the foil's low thickness compared to the grain dimension causes significant interfacial stresses which promote the 100 texture to be the lowest energy state. ${ }^{25}$

Conversely, annealing with a cap was found to increase the chance of producing 111 textures (Fig. 3(a)) which is the lowest energy state of an infinite $\mathrm{Cu}$ crystal. ${ }^{26-28}$ This observation is important because growth on 111 textures is expected to produce higher quality graphene ${ }^{2}$ but was previously only achieved on expensive single-crystalline substrates.

We furthermore note that capped annealing can also produce large areas of exotic textures, such has 121 and 102 (Fig. 3(a)), which shows the potential of our approach for engineering the atomic orientation of metal substrates.

An improved strain relaxation is expected to significantly affect the morphology of the $\mathrm{Cu}$ surface. Theoretical studies found a linear relation between strain and surface roughness due to elastic deformation and predicted an increase of this effect with larger grain sizes. ${ }^{29}$

Atomic force micrographs indeed show a smoother surface for relaxed samples after capped annealing compared to strained samples after uncapped annealing (Fig. 3(b)). This result confirms the formation of an effective interface between cap and sample.

Moreover, we observe a direct correlation between the XRD peak position of copper and its roughness (Fig. 3(c)) which supports our model that strain is the controlling factor for the $\mathrm{Cu}$ roughness. This result highlights the potential of strain relaxation to reduce the roughness of the substrate.

The here presented ability to control the Cu morphology was subsequently applied enhance the performance of graphene films grown on it.

For this purpose $\mathrm{Cu}$ was first annealed in the described capped or uncapped conditions. All of the thus produced $\mathrm{Cu}$ foils were then subjected to CVD at the same time to limit the sample-to-sample variation. CVD for all samples was carried out using enclosure growth as reported previously. ${ }^{21}$

We observe that graphene grown on relaxed $\mathrm{Cu}$ foil shows a lower grain density and an increased grain size compared to $\mathrm{Cu}$ foil after uncapped annealing (Fig. 4(a)). This change is ascribed to a lower roughness of relaxed $\mathrm{Cu}$ that results in
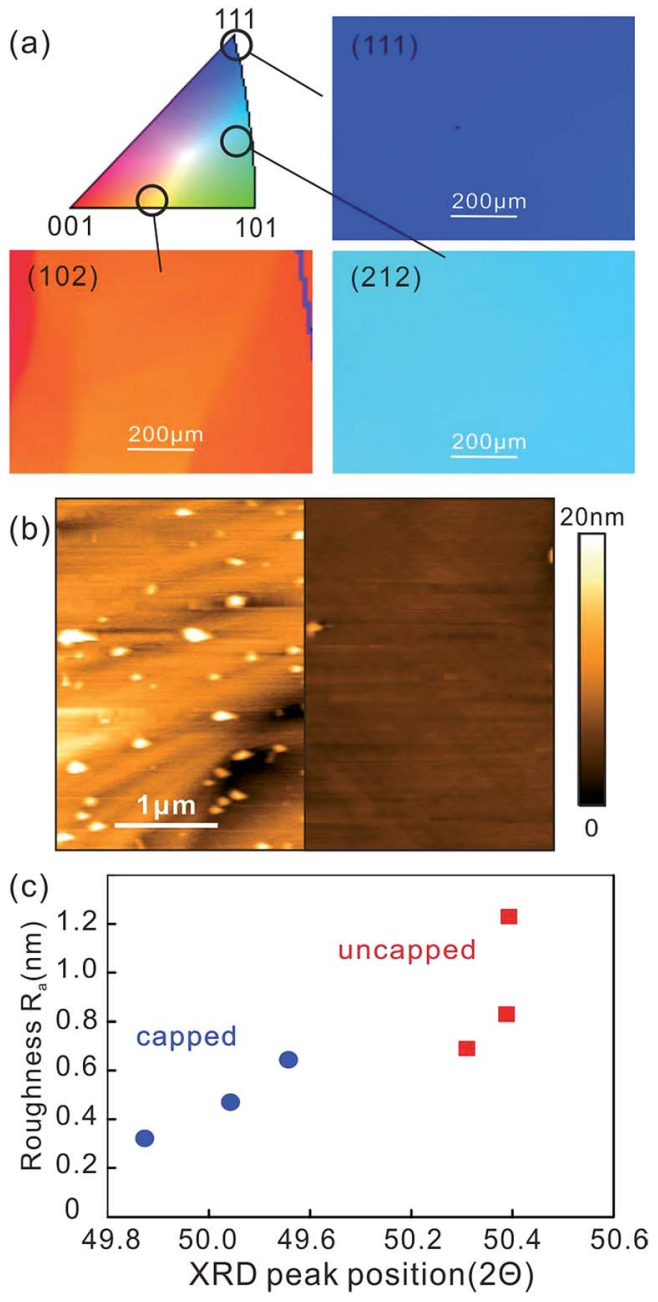

Fig. 3 (a) EBSD images of 3 representative samples that were subjected to 8 hour capped annealing under identical conditions and their position in the color legend, (b) representative AFM images after $8 \mathrm{~h}$ annealing for uncapped (left) and capped $\mathrm{Cu}$ (right) (c) average XRD Cu (200) peak position vs. Cu roughness.

a decreasing concentration of suitable protrusions which can act as nucleation seeds. ${ }^{30}$ Additionally, the increased grain size results in a lower density of grain boundaries which have been associated with structural defects in the graphene lattice. ${ }^{31}$ Indeed Raman analysis shows a lower defectiveness of graphene grown on cap-annealed $\mathrm{Cu}$ as indicated by a lower average $I_{\mathrm{D}} / I_{\mathrm{G}}$ ratio (Fig. 4(b) $)^{32}$ (for detailed characterization of graphene grown on cap annealed or uncapped $\mathrm{Cu}$ foil refer to ESI Fig. S2 $\dagger$ ). This result was confirmed by FIFE analysis ${ }^{33}$ which shows a higher coverage for capped Cu annealing (ESI Fig. S3†).

Finally, the carrier mobility of graphene grown on differently annealed $\mathrm{Cu}$ foils was investigated (Fig. 4(c)). We find a clear trend of an improvement of mobility with annealing duration which corroborates the importance of an improved catalyst morphology. Furthermore graphene grown on $12 \mathrm{~h}$ capannealed $\mathrm{Cu}$ exhibited an increase in average mobility of $25 \%$ which illustrates the potential of our approach. 
(a)

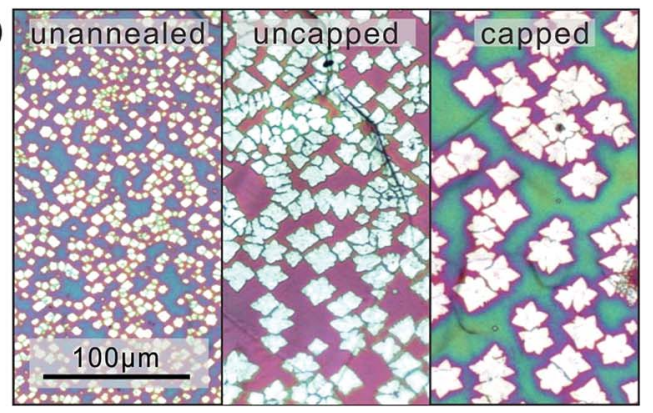

(b)

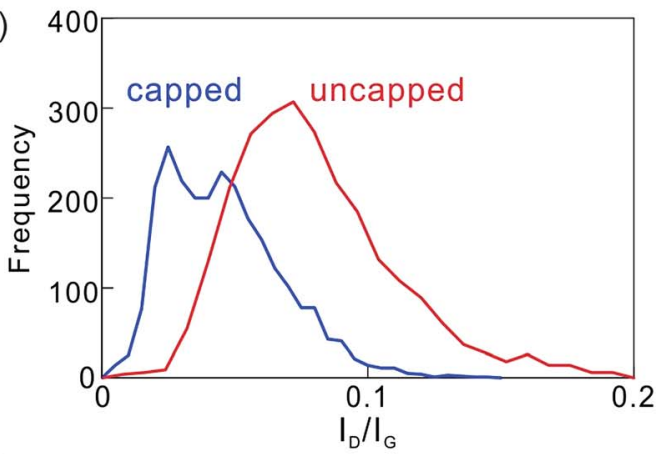

(c)

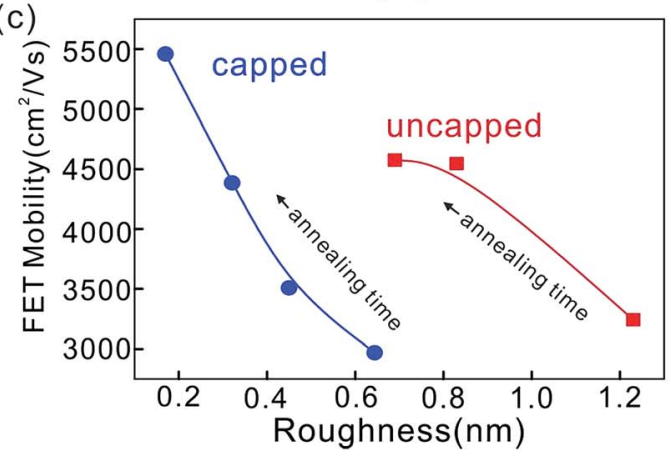

Fig. 4 (a) Representative OM images of graphene grown on differently annealed $\mathrm{Cu}$ foil, (b) histograms of Raman $I_{\mathrm{D}} / /_{\mathrm{G}}$ ratio for graphene grown on Cu foil after capped/uncapped annealing, (c) graphene FET mobility vs. roughness for capped and uncapped Cu foil after different annealing durations.

\section{Conclusions}

In conclusion, we have found that capped annealing can help decreasing the interfacial stress of copper foil. This effect enhances its grain growth rate and lowers the crystalline strain as confirmed by EBSD and XRD. Capped annealing was shown to reduce the roughness of copper which helps to grow graphene in high quality.

\section{Acknowledgements}

YP Hsieh and M. Hofmann acknowledge financial support from Applied Materials, Inc., the Ministry of Science and Technology, and the Industrial Technology Research Institute of Taiwan.

\section{References}

1 K. S. Novoselov, V. I. Falko, L. Colombo, P. R. Gellert, M. G. Schwab and K. Kim, Nature, 2012, 490, 192-200.
2 Y. Ogawa, B. Hu, C. M. Orofeo, M. Tsuji, K.-i. Ikeda, S. Mizuno, H. Hibino and H. Ago, J. Phys. Chem. Lett., 2012, 3, 219-226.

3 J. D. Wood, S. W. Schmucker, A. S. Lyons, E. Pop and J. W. Lyding, Nano Lett., 2011, 11, 4547-4554.

4 L. Zhao, K. T. Rim, H. Zhou, R. He, T. F. Heinz, A. Pinczuk, G. W. Flynn and A. N. Pasupathy, Solid State Commun., 2011, 151, 509-513.

5 A. T. Murdock, A. Koos, T. B. Britton, L. Houben, T. Batten, T. Zhang, A. J. Wilkinson, R. E. Dunin-Borkowski, C. E. Lekka and N. Grobert, ACS Nano, 2013, 7, 1351-1359.

6 H. Wang, G. Wang, P. Bao, S. Yang, W. Zhu, X. Xie and W.-J. Zhang, J. Am. Chem. Soc., 2012, 134, 3627-3630.

7 Z. Yan, J. Lin, Z. Peng, Z. Sun, Y. Zhu, L. Li, C. Xiang, E. L. Samuel, C. Kittrell and J. M. Tour, ACS Nano, 2012, 6, 9110-9117.

8 Y. A. Wu, Y. Fan, S. Speller, G. L. Creeth, J. T. Sadowski, K. He, A. W. Robertson, C. S. Allen and J. H. Warner, ACS Nano, 2012, 6, 5010-5017.

9 D. Geng, B. Wu, Y. Guo, L. Huang, Y. Xue, J. Chen, G. Yu, L. Jiang, W. Hu and Y. Liu, Proc. Natl. Acad. Sci. U. S. A., 2012, 109, 7992-7996.

10 P. Y. Huang, C. S. Ruiz-Vargas, A. M. van der Zande, W. S. Whitney, M. P. Levendorf, J. W. Kevek, S. Garg, J. S. Alden, C. J. Hustedt, Y. Zhu, J. Park, P. L. McEuen and D. A. Muller, Nature, 2011, 469, 389-392.

11 H. Ago, Y. Ogawa, M. Tsuji, S. Mizuno and H. Hibino, J. Phys. Chem. Lett., 2012, 3, 2228-2236.

12 Q. Yu, L. A. Jauregui, W. Wu, R. Colby, J. Tian, Z. Su, H. Cao, Z. Liu, D. Pandey, D. Wei, T. F. Chung, P. Peng, N. P. Guisinger, E. A. Stach, J. Bao, S.-S. Pei and Y. P. Chen, Nat. Mater., 2011, 10, 443-449.

13 M. D. S. Lakshad Wimalananda, J.-K. Kim and J.-M. Lee, Carbon, 2016, 108, 127-134.

14 K. P. Sharma, S. M. Shinde, M. S. Rosmi, S. Sharma, G. Kalita and M. Tanemura, J. Mater. Sci., 2016, 51, 7220-7228.

15 L. Brown, E. B. Lochocki, J. Avila, C.-J. Kim, Y. Ogawa, R. W. Havener, D.-K. Kim, E. J. Monkman, D. E. Shai and H. I. Wei, Nano Lett., 2014, 14, 5706-5711.

16 L. Lu, M. Sui and K. Lu, Acta Mater., 2001, 49, 4127-4134.

17 G. K. Williamson and R. E. Smallman, Philos. Mag., 1956, 1, 34-46.

18 S. Schmidt, S. F. Nielsen, C. Gundlach, L. Margulies, X. Huang and D. J. Jensen, Science, 2004, 305, 229-232.

19 R. E. Reed-Hill and R. Abbaschian, Physical metallurgy principles, 1973.

20 A. Vattré, T. Jourdan, H. Ding, M. C. Marinica and M. J. Demkowicz, Nat. Commun., 2016, 7, 10424.

21 Y. P. Hsieh, Y. J. Chiu and M. Hofmann, Nanoscale, 2015, 7, 19403-19407.

22 D. Nezich, A. Reina and J. Kong, Nanotechnology, 2011, 23, 015701.

23 D. L. Duong, G. H. Han, S. M. Lee, F. Gunes, E. S. Kim, S. T. Kim, H. Kim, Q. H. Ta, K. P. So and S. J. Yoon, Nature, 2012, 490, 235-239.

24 S. K. Ganapathi, D. M. Owen and A. H. Chokshi, Scr. Metall. Mater., 1991, 25, 2699-2704. 
25 N. J. Park, D. P. Field, M. M. Nowell and P. R. Besser, J. Electron. Mater., 2005, 34, 1500-1508.

26 C. V. Thompson and R. Carel, J. Mech. Phys. Solids, 1996, 44, 657-673.

27 J. M. E. Harper, C. Cabral, P. C. Andricacos, L. Gignac, I. C. Noyan, K. P. Rodbell and C. K. Hu, J. Appl. Phys., 1999, 86, 2516.

28 S.-C. Chang, J.-M. Shieh, B.-T. Dai, M.-S. Feng and Y.-H. Li, J. Electrochem. Soc., 2002, 149, G535.

29 A. Perron, I. O. Politano and V. Vignal, Grain size, stress and surface roughness, Wiley, Chichester, ROYAUME-UNI, 2008.
$30 \mathrm{H}$. Ya-Ping, C. Yi-Hung, T. He-Guang and H. Mario, Nanotechnology, 2016, 27, 105602.

31 H. Zhou, W. J. Yu, L. Liu, R. Cheng, Y. Chen, X. Huang, Y. Liu, Y. Wang, Y. Huang and X. Duan, Nat. Commun., 2013, 4, 2096.

32 M. S. Dresselhaus, A. Jorio, M. Hofmann, G. Dresselhaus and R. Saito, Nano Lett., 2010, 10, 751-758.

33 M. Hofmann, Y. C. Shin, Y.-P. Hsieh, M. S. Dresselhaus and J. Kong, Nano Res., 2012, 5, 504-511. 